\title{
Kalman controllability decompositions for differential-algebraic systems
}

\author{
Thomas Berger ${ }^{\mathrm{a}}$, Stephan Trenn ${ }^{\mathrm{b}}$ \\ ${ }^{a}$ Fachbereich Mathematik, Universität Hamburg, Bundesstraße 55, 20146 Hamburg, Germany \\ ${ }^{b}$ Fachbereich Mathematik, Technische Universität Kaiserslautern, Postfach 3049, 67653 Kaiserslautern, Germany
}

\begin{abstract}
We study linear differential-algebraic equations and investigate decompositions with respect to controllability properties. We show that the augmented Wong sequences can be exploited for a transformation of the system into a Kalman controllability decomposition (KCD). The KCD decouples the system into a completely controllable part, an uncontrollable part given by an ordinary differential equation and an inconsistent part, which is controllable in the behavioral sense but contains no completely controllable part. This decomposition improves a known KCD from a behavioral point of view. We conclude the paper with some features of the KCD in the case of regular systems.
\end{abstract}

Keywords: differential-algebraic systems; controllability; Kalman decomposition; Wong sequences.

\section{Introduction}

We consider linear constant coefficient differential-algebraic equations (DAEs) of the form

$$
E \dot{x}(t)=A x(t)+B u(t)
$$

where $E, A \in \mathbb{R}^{l \times n}, B \in \mathbb{R}^{l \times m}$. The set of system models given by (1) is denoted by $\Sigma_{m}^{l \times n}$ and we write $(E, A, B) \in \Sigma_{m}^{l \times n}$. DAEs of the form (1) naturally occur when modeling dynamical systems subject to algebraic constraints; for a further motivation we refer to $[8,10,12]$ and the references therein. The DAE $(E, A, B)$ is called regular if $l=n$ and $\operatorname{det}(s E-A) \in \mathbb{R}[s] \backslash\{0\}$; otherwise it is called singular. We stress at this point that our main result concerning the Kalman controllability decomposition (KCD) holds for the regular as well as for the singular case.

There is a canonical equivalence notion for DAEs in $\Sigma_{m}^{l \times n}$ given by

$$
(E, A, B) \cong(\tilde{E}, \tilde{A}, \tilde{B}) \quad: \Longleftrightarrow \quad \exists S \in \mathbf{G L}_{l}, T \in \mathbf{G L}_{n}:(S E T, S A T, S B)=(\tilde{E}, \tilde{A}, \tilde{B}),
$$

where $\mathbf{G L}_{k}$ denotes the space of invertible real-valued $k \times k$ matrices; $\cong$ is also often called system equivalence, first studied by Rosenbrock [13]. If we want to highlight the involved transformation matrices $S$ and $T$ we also write $\stackrel{S, T}{\cong}$ instead of $\cong$. The desired KCD, presented later, is a special representative of the corresponding equivalence class where controllability properties can easily be read off.

The function $u: \mathbb{R} \rightarrow \mathbb{R}^{m}$ is usually called input of the system, although one should keep in mind, that in the singular case $u$ might be constrained and some of the state variables can play the role of an input.

The tuple $(x, u): \mathbb{R} \rightarrow \mathbb{R}^{n} \times \mathbb{R}^{m}$ is said to be a solution of (1) if, and only if, it belongs to the behavior of (1):

$$
\mathfrak{B}_{(E, A, B)}:=\left\{(x, u) \in \mathscr{W}_{\text {loc }}^{1}\left(\mathbb{R} \rightarrow \mathbb{R}^{n}\right) \times \mathscr{L}_{\text {loc }}^{1}\left(\mathbb{R} \rightarrow \mathbb{R}^{m}\right) \mid(x, u) \text { satisfies (1) for almost all } t \in \mathbb{R}\right\},
$$

where $\mathscr{L}_{\text {loc }}^{1}$ and $\mathscr{W}_{\text {loc }}^{1}$ denote the space of locally (Lebesgue) integrable or weakly differentiable functions with locally integrable derivatives (see [1, Chap. 1]), respectively. The equivalence of DAEs translates to an equivalence of

Email addresses: thomas.berger@uni-hamburg.de (Thomas Berger), trenn@mathematik.uni-kl.de (Stephan Trenn) 
solutions as follows:

$$
(E, A, B) \stackrel{S, T}{\cong}(\tilde{E}, \tilde{A}, \tilde{B}) \quad \Longrightarrow \quad\left[(x, u) \in \mathfrak{B}_{(E, A, B)} \Leftrightarrow\left(T^{-1} x, u\right) \in \mathfrak{B}_{(\tilde{E}, \tilde{A}, \tilde{B})}\right]
$$

in particular the input is not altered.

Note that it is possible to consider a slightly larger solution space by only requiring that $x \in \mathscr{L}_{\text {loc }}^{1}$ and $E x \in \mathscr{W}_{\text {loc }}^{1}$, see [3]; in particular, it is not necessary to assume that $x$ is continuous. However, this leads to some technical difficulties when studying (complete) controllability and therefore we restrict our attention to the above solution concept.

In the present paper we are interested in a KCD for general DAEs of the form (1). Recall the well-known result that for linear ordinary differential equations (ODEs) of the form

$$
\dot{x}(t)=A x(t)+B u(t)
$$

the $\mathrm{KCD}$ is given by

$$
\dot{z}(t)=\left[\begin{array}{cc}
A_{11} & A_{12} \\
0 & A_{22}
\end{array}\right] z(t)+\left[\begin{array}{c}
B_{1} \\
0
\end{array}\right] u(t),
$$

where $x \mapsto T z$ is a suitable coordinate transformation such that the ODE $\dot{z}_{1}(t)=A_{11} z_{1}(t)+B_{1} u(t)$ is controllable. In particular, the KCD separates the ODE into a controllable and an uncontrollable part. At first glance, a satisfying generalization of (2) is also available for DAEs (even in the singular case), see [5, Thm. 7.1] (which is based on a result for the discrete time case in [2]): There exist invertible matrices $S$ and $T$ such that

$$
(E, A, B) \stackrel{S, T}{\cong}\left(\left[\begin{array}{cc}
E_{11} & E_{12} \\
0 & E_{22}
\end{array}\right],\left[\begin{array}{cc}
A_{11} & A_{12} \\
0 & A_{22}
\end{array}\right],\left[\begin{array}{c}
B_{1} \\
0
\end{array}\right]\right)
$$

where the DAE $\left(E_{11}, A_{11}, B_{1}\right)$ is completely controllable (see the forthcoming Definition 2.1) and the only reachable state from the origin for the DAE $\left(E_{22}, A_{22}, 0\right)$ is the origin itself. Seemingly, we again have a decomposition into a controllable and an uncontrollable part. However, in the behavioral approach (see e.g. [11]) the trivial DAE $0=x$ given by $(0, I, 0)$ is controllable (because any two trajectories can be concatenated within the behavior), but the above KCD would only consist of the uncontrollable part. This is an unsatisfactory situation and is due to the fact, that for DAEs (both regular and singular) certain states are inconsistent and it doesn't really make sense to label those controllable or uncontrollable. We therefore propose the following more detailed KCD:

$$
(S E T, S A T, S B)=\left(\left[\begin{array}{ccc}
E_{11} & E_{12} & E_{13} \\
0 & E_{22} & E_{23} \\
0 & 0 & E_{33}
\end{array}\right],\left[\begin{array}{ccc}
A_{11} & A_{12} & A_{13} \\
0 & A_{22} & A_{23} \\
0 & 0 & A_{33}
\end{array}\right],\left[\begin{array}{c}
B_{1} \\
0 \\
0
\end{array}\right]\right),
$$

where, as before, $S$ and $T$ are invertible matrices and the DAE given by $\left(E_{11}, A_{11}, B_{1}\right)$ is completely controllable. Furthermore, $E_{22}$ is invertible and the DAE $\left(E_{33}, A_{33}, 0\right)$ is such that it only has the trivial solution. Hence, we now have the decomposition into a (completely) controllable part, a classical uncontrollable part (given by an ODE) and an inconsistent part (which is behavioral controllable but contains no completely controllable part). We believe that this KCD is much more adequate for the analysis of DAEs as it takes into account the special DAE feature of possible inconsistent states which play a special role with respect to controllability. When restricting the attention to the case of regular DAEs, we obtain a further decomposition of the completely controllable part into a classical controllable part (given by a controllable ODE) and an instantaneously controllable part (corresponding to a controllable "pure" DAE).

The paper is organized as follows: In Section 2 we introduce the concepts of complete and behavioral controllability considered in the present paper. We also recall the augmented Wong sequences as the crucial geometric tool for our investigations and some connections of these sequences with the controllability concepts, the reachable space and the space of consistent initial values. The $\mathrm{KCD}$ for singular $(E, A, B)$ is proved in Section 3 and uniqueness of the decomposition with respect to the equivalence $\cong$ is discussed. Finally, Section 4 is devoted to the case of regular systems and some features of the KCD are highlighted. In particular, the connection between the augmented Wong 
sequences and the original Wong sequences $(B=0)$ is shown; this is also illustrated in Figure 1.

\section{Controllability notions}

We recall the concepts of complete controllability and controllability in the behavioral sense and their geometric characterizations in terms of augmented Wong sequences. Our presentation follows mostly the survey [5].

Definition 2.1 (Controllability concepts). A system $(E, A, B) \in \Sigma_{m}^{l \times n}$ is called

(i) completely controllable if, and only if,

$$
\forall x_{0}, x_{f} \in \mathbb{R}^{n} \exists t_{f}>0 \exists(x, u) \in \mathfrak{B}_{(E, A, B)}: \quad x(0)=x_{0} \wedge x\left(t_{f}\right)=x_{f},
$$

i.e., it is possible to control the state $x(\cdot)$ from any given initial value $x_{0}$ to any final value $x_{f}$.

(ii) controllable in the behavioral sense if, and only if,

$$
\forall\left(x_{1}, u_{1}\right),\left(x_{2}, u_{2}\right) \in \mathfrak{B}_{(E, A, B)} \exists t_{f}>0 \exists(x, u) \in \mathfrak{B}_{(E, A, B)}:(x(t), u(t))= \begin{cases}\left(x_{1}(t), u_{1}(t)\right), & \text { if } t<0, \\ \left(x_{2}(t), u_{2}(t)\right), & \text { if } t>t_{f},\end{cases}
$$

i.e., it is possible to connect any two feasible trajectories via a third feasible trajectory.

Both controllability notions are equivalent for ODEs; however, for DAEs (singular as well as regular) complete controllability is stronger than behavioral controllability as the latter does not require that the reachable space (see the forthcoming Definition 2.3) is the whole space; it is defined within the context of the behavioral approach [11] and hence respects the underlying algebraic constraints.

In order to geometrically characterize controllability, the augmented Wong sequences are an important tool (see [5] and the references therein) and are defined as follows:

$$
\begin{array}{lll}
\mathscr{V}_{(E, A, B)}^{0}:=\mathbb{R}^{n}, & \mathscr{V}_{(E, A, B)}^{i+1}:=A^{-1}\left(E^{\mathscr{V}} \mathcal{V}_{(E, A, B)}^{i}+\operatorname{im} B\right) \subseteq \mathbb{R}^{n}, & \mathscr{V}_{(E, A, B)}^{*}:=\bigcap_{i \in \mathbb{N}_{0}} \mathscr{V}_{(E, A, B)}^{i}, \\
\mathscr{W}_{(E, A, B)}^{0}:=\{0\}, & \mathscr{W}_{(E, A, B)}^{i+1}:=E^{-1}\left(A \mathscr{W}_{(E, A, B)}^{i}+\operatorname{im} B\right) \subseteq \mathbb{R}^{n}, & \mathscr{W}_{(E, A, B)}^{*}:=\bigcup_{i \in \mathbb{N}_{0}} \mathscr{W}_{(E, A, B)}^{i} .
\end{array}
$$

The sequences $\left(\mathscr{V}_{(E, A, B)}^{i}\right)_{i \in \mathbb{N}}$ and $\left(\mathscr{W}_{(E, A, B)}^{i}\right)_{i \in \mathbb{N}}$ are called augmented Wong sequences since they are based on the Wong sequences $(B=0)$ used in $[4,6,7]$ and which have their origin in WONG [17] who was the first using both sequences (with $B=0$ ) for the analysis of matrix pencils.

The augmented Wong sequences allow a characterization of the controllability concepts as follows.

Lemma 2.2 (Geometric criteria for controllability [5]). Consider $(E, A, B) \in \Sigma_{m}^{l \times n}$ and the limits $\mathscr{V}_{(E, A, B)}^{*}$ and $\mathscr{W}_{(E, A, B)}^{*}$ of the augmented Wong sequences. Then $(E, A, B)$ is

(a) completely controllable if, and only if, $\mathscr{V}_{(E, A, B)}^{*} \cap \mathscr{W}_{(E, A, B)}^{*}=\mathbb{R}^{n}$;

(b) controllable in the behavioral sense if, and only if, $\mathscr{V}_{(E, A, B)}^{*} \subseteq \mathscr{W}_{(E, A, B)}^{*}$.

Before we can state the Kalman controllability decomposition, we also need the notion of the reachable space which is crucial for the proof of the decomposition.

Definition 2.3 (Reachable space, for details see [5]). For $(E, A, B) \in \Sigma_{m}^{l \times n}$ the reachable space is defined as

$$
\mathscr{R}_{(E, A, B)}:=\left\{x_{f} \in \mathbb{R}^{n} \mid \exists t_{f}>0 \exists(x, u) \in \mathfrak{B}_{(E, A, B)}: x(0)=0 \wedge x\left(t_{f}\right)=x_{f}\right\}
$$

Note that any reachable state $x_{f} \in \mathbb{R}^{n}$ can be reached from the origin in arbitrary time $t_{f}>0$ (i.e., in the definition above " $\exists t_{f}>0$ " can be replaced by " $\forall t_{f}>0$ "). 
Remark 2.4. In [5] it is shown that $\mathscr{R}_{(E, A, B)}=\mathscr{V}_{(E, A, B)}^{*} \cap \mathscr{W}_{(E, A, B)}^{*}$, hence complete controllability can also be characterized by the intuitive condition $\mathscr{R}_{(E, A, B)}=\mathbb{R}^{n}$. Furthermore, it is proved in [5] that the space of consistent initial values is given by $\mathscr{V}_{(E, A, B)}^{*}$, i.e., $\left\{x_{0} \in \mathbb{R}^{n} \mid \exists(x, u) \in \mathfrak{B}_{(E, A, B)}: x(0)=x_{0}\right\}=\mathscr{V}_{(E, A, B)}^{*}$.

\section{Kalman controllability decomposition}

In this section we present our main result about a generalized Kalman controllability decomposition (KCP) which respects the special features of DAEs. In fact, this decoupling can be obtained form the augmented Wong sequences of the original system, which also yield a simple procedure to obtain the basis transformation. In view of Remark 2.4 , this basis transformation can intuitively be obtained from Lemma 2.2: the subspace $\mathscr{V}_{(E, A, B)}^{*} \cap \mathscr{W}_{(E, A, B)}^{*}$ yields the completely controllable part, any complement of $\mathscr{V}_{(E, A, B)}^{*} \cap \mathscr{W}_{(E, A, B)}^{*}$ in $\mathscr{V}_{(E, A, B)}^{*}$ yields an uncontrollable part (since $\mathscr{V}_{(E, A, B)}^{*} \subseteq \mathscr{W}_{(E, A, B)}^{*}$ is equivalent to $\left.\mathscr{V}_{(E, A, B)}^{*} \cap \mathscr{W}_{(E, A, B)}^{*}=\mathscr{V}_{(E, A, B)}^{*}\right)$, and any complement of $\mathscr{V}_{(E, A, B)}^{*}$ yields a behavioral controllable part that is not completely controllable (i.e., a subspace of inconsistent initial values).

Theorem 3.1 (Kalman controllability decomposition). Consider $(E, A, B) \in \Sigma_{m}^{l \times n}$ and the limits $\mathscr{V}_{(E, A, B)}^{*}$ and $\mathscr{W}_{(E, A, B)}^{*}$ of the augmented Wong sequences. Choose any full rank matrices $R_{1} \in \mathbb{R}^{n \times n_{1}}, P_{1} \in \mathbb{R}^{n \times n_{2}}, Q_{1} \in \mathbb{R}^{n \times n_{3}}, R_{2} \in \mathbb{R}^{l \times l_{1}}, P_{2} \in$ $\mathbb{R}^{l \times l_{2}}, Q_{2} \in \mathbb{R}^{l \times l_{3}}$ such that

$$
\begin{aligned}
\operatorname{im} R_{1} & =\mathscr{V}_{(E, A, B)}^{*} \cap \mathscr{W}_{(E, A, B)}^{*}, & \operatorname{im} R_{2} & =\left(E \mathscr{V}_{(E, A, B)}^{*}+\operatorname{im} B\right) \cap\left(A \mathscr{W}_{(E, A, B)}^{*}+\operatorname{im} B\right), \\
\operatorname{im} R_{1} \oplus \operatorname{im} P_{1} & =\mathscr{V}_{(E, A, B)}^{*}, & \operatorname{im} R_{2} \oplus \operatorname{im} P_{2} & =E \mathscr{V}_{(E, A, B)}^{*}+\operatorname{im} B, \\
\operatorname{im}\left[R_{1}, P_{1}\right] \oplus \operatorname{im} Q_{1} & =\mathbb{R}^{n}, & \operatorname{im}\left[R_{2}, P_{2}\right] \oplus \operatorname{im} Q_{2} & =\mathbb{R}^{l}
\end{aligned}
$$

Then $T:=\left[R_{1}, P_{1}, Q_{1}\right] \in \mathbf{G L}_{n}$ and $S:=\left[R_{2}, P_{2}, Q_{2}\right]^{-1} \in \mathbf{G L}_{l}$ transform $(E, A, B)$ into $K C D$ :

$$
(E, A, B) \stackrel{S, T}{\cong}\left(\left[\begin{array}{ccc}
E_{11} & E_{12} & E_{13} \\
0 & E_{22} & E_{23} \\
0 & 0 & E_{33}
\end{array}\right],\left[\begin{array}{ccc}
A_{11} & A_{12} & A_{13} \\
0 & A_{22} & A_{23} \\
0 & 0 & A_{33}
\end{array}\right],\left[\begin{array}{c}
B_{1} \\
0 \\
0
\end{array}\right]\right)
$$

where

(i) $\left(E_{11}, A_{11}, B_{1}\right) \in \sum_{m}^{l_{1} \times n_{1}}$ with $l_{1}=\operatorname{rk}\left[E_{11}, B_{1}\right] \leq n_{1}+m$ is completely controllable,

(ii) $\left(E_{22}, A_{22}, 0\right) \in \Sigma_{m}^{l_{2} \times n_{2}}$ with $l_{2}=n_{2}$ and $E_{22}$ is invertible,

(iii) $\left(E_{33}, A_{33}, 0\right) \in \Sigma_{m}^{l_{3} \times n_{3}}$ with $l_{3} \geq n_{3}$ satisfies $\mathrm{rk}_{\mathbb{C}}\left(\lambda E_{33}-A_{33}\right)=n_{3}$ for all $\lambda \in \mathbb{C}$.

Proof. Step 1: First observe that the subspace inclusions

$$
\begin{aligned}
E\left(\mathscr{V}_{(E, A, B)}^{*} \cap \mathscr{W}_{(E, A, B)}^{*}\right) & \subseteq\left(E_{(E, A, B)}^{*}+\operatorname{im} B\right) \cap\left(A \mathscr{W}_{(E, A, B)}^{*}+\operatorname{im} B\right), \\
A\left(\mathscr{V}_{(E, A, B)}^{*} \cap \mathscr{W}_{(E, A, B)}^{*}\right) & \subseteq\left(E \mathscr{V}_{(E, A, B)}^{*}+\operatorname{im} B\right) \cap\left(A \mathscr{W}_{(E, A, B)}^{*}+\operatorname{im} B\right), \\
E \mathscr{V}_{(E, A, B)}^{*} & \subseteq E \mathscr{V}_{(E, A, B)}^{*}+\operatorname{im} B, \\
A \mathscr{V}_{(E, A, B)}^{*} & \subseteq E \mathscr{V}_{(E, A, B)}^{*}+\operatorname{im} B,
\end{aligned}
$$

imply existence of $E_{11}, \ldots, E_{33}$ and $A_{11}, \ldots, A_{33}$ such that

$$
\begin{aligned}
E R_{1} & =R_{2} E_{11}, & A R_{1} & =R_{2} A_{11}, \\
E P_{1} & =R_{2} E_{12}+P_{2} E_{22}, & A P_{1} & =R_{2} A_{12}+P_{2} A_{22}, \\
E Q_{1} & =R_{2} E_{13}+P_{2} E_{23}+Q_{2} E_{33}, & A Q_{1} & =R_{2} A_{13}+P_{2} A_{23}+Q_{2} A_{33} .
\end{aligned}
$$

Since $\operatorname{im} B \subseteq\left(E \mathscr{V}^{*}+\operatorname{im} B\right) \cap\left(A \mathscr{W}^{*}+\operatorname{im} B\right)=\operatorname{im} R_{2}$, there exists $B_{1} \in \mathbb{R}^{l_{1} \times m}$ such that $B=R_{2} B_{1}$. All these relations together yield the decomposition (3). 
Step 2: We show (i) by proceeding in several steps.

Step $2 a$ : We show that $\left(E_{11}, A_{11}, B_{1}\right)$ is completely controllable.

By Remark 2.4 we have for the reachable space that

$$
\mathscr{R}_{(E, A, B)}=\mathscr{V}_{(E, A, B)}^{*} \cap \mathscr{W}_{(E, A, B)}^{*}=\operatorname{im} R_{1}=T\left(\mathbb{R}^{n_{1}} \times\{0\}^{n_{2}+n_{3}}\right) .
$$

Since any $(z, u) \in \mathfrak{B}_{(S E T, S A T, S B)}$ with $z=\left(z_{1}^{\top}, z_{2}^{\top}, z_{3}^{\top}\right)^{\top} \in \mathscr{W}_{\text {loc }}^{1}\left(\mathbb{R} \rightarrow \mathbb{R}^{n}\right)$ and $z(0)=0$ satisfies

$$
\begin{aligned}
E_{11} \dot{z}_{1}(t)+E_{12} \dot{z}_{2}(t)+E_{13} \dot{z}_{3}(t) & =A_{13} z_{1}(t)+A_{12} z_{2}(t)+A_{13} z_{3}(t)+B_{1} u(t) \\
E_{22} \dot{z}_{2}(t)+E_{23} \dot{z}_{3}(t) & =A_{22} z_{2}(t)+A_{23} z_{3}(t) \\
E_{33} \dot{z}_{3}(t) & =A_{33} z_{3}(t)
\end{aligned}
$$

the assumption that

$$
\forall t \geq 0: z(t) \in \mathscr{R}_{(S E T, S A T, S B)}=T^{-1} \mathscr{R}_{(E, A, B)}=\mathbb{R}^{n_{1}} \times\{0\}^{n_{2}+n_{3}}
$$

leads to $z_{2} \equiv 0$ and $z_{3} \equiv 0$. This implies that

$$
\mathscr{R}_{(S E T, S A T, S B)}=\mathscr{R}_{\left(E_{11}, A_{11}, B_{1}\right)} \times\{0\}^{n_{2}+n_{3}}
$$

and hence we find $\mathscr{R}_{\left(E_{11}, A_{11}, B_{1}\right)}=\mathbb{R}^{n_{1}}$, which according to Remark 2.4 is equivalent to $\left(E_{11}, A_{11}, B_{1}\right) \in \Sigma_{m}^{l_{1} \times n_{1}}$ being completely controllable.

Step 2b: We show $E\left(\mathscr{V}_{(E, A, B)}^{*} \cap \mathscr{W}_{(E, A, B)}^{*}\right)+\operatorname{im} B=\left(E \mathscr{V}_{(E, A, B)}^{*}+\operatorname{im} B\right) \cap\left(A \mathscr{W}_{(E, A, B)}^{*}+\operatorname{im} B\right)$.

The inclusion " $\subseteq$ " was already observed in Step 1. For " $\supseteq$ " let $x \in\left(E_{(E, A, B)}^{*}+\operatorname{im} B\right) \cap\left(A \mathscr{W}_{(E, A, B)}^{*}+\operatorname{im} B\right)$, i.e., $x=E v+b_{1}=A w+b_{2}$ for some $v \in \mathscr{V}_{(E, A, B)}^{*}, w \in \mathscr{W}_{(E, A, B)}^{*}, b_{1}, b_{2} \in \operatorname{im} B$. Then

$$
v \in E^{-1}\left\{A w+b_{2}-b_{1}\right\} \subseteq E^{-1}\left(A \mathscr{W}_{(E, A, B)}^{*}+\operatorname{im} B\right)=\mathscr{W}_{(E, A, B)}^{*} .
$$

Therefore, $v \in \mathscr{V}_{(E, A, B)}^{*} \cap \mathscr{W}_{(E, A, B)}^{*}$ and hence $x=E v+b_{1} \in E\left(\mathscr{V}_{(E, A, B)}^{*} \cap \mathscr{W}_{(E, A, B)}^{*}\right)+\operatorname{im} B$.

Step $2 c$ : We show that $\operatorname{rk}\left[E_{11}, B_{1}\right]=l_{1}$.

Since $\operatorname{im} R_{2} E_{11}=\operatorname{im} E R_{1}=E\left(\mathscr{V}_{(E, A, B)}^{*} \cap \mathscr{W}_{(E, A, B)}^{*}\right)$ we find that

$$
\operatorname{im} R_{2}\left[E_{11}, B_{1}\right]=E\left(\mathscr{V}_{(E, A, B)}^{*} \cap \mathscr{W}_{(E, A, B)}^{*}\right)+\operatorname{im} B=\operatorname{im} R_{2},
$$

where the latter equality follows from Step $2 \mathrm{~b}$. Therefore, full column rank of $R_{2}$ implies $\operatorname{im}\left[E_{11}, B_{1}\right]=\mathbb{R}^{l_{1}}$ and hence $l_{1}=\operatorname{rk}\left[E_{11}, B_{1}\right] \leq \mathrm{rk} E_{11}+\mathrm{rk} B_{1} \leq n_{1}+m$.

Step 3: We show (ii).

Clearly,

Step 3a: We show that $\left(E\left(\mathscr{V}_{(E, A, B)}^{*} \cap \mathscr{W}_{(E, A, B)}^{*}\right)+\operatorname{im} B\right) \oplus \operatorname{im} E P_{1}=E_{\mathscr{V}_{(E, A, B)}^{*}}^{*}+\operatorname{im} B$.

$$
E \mathscr{V}_{(E, A, B)}^{*}+\operatorname{im} B=E\left(\left(\mathscr{V}_{(E, A, B)}^{*} \cap \mathscr{W}_{(E, A, B)}^{*}\right) \oplus \operatorname{im} P_{1}\right)+\operatorname{im} B=E\left(\mathscr{V}_{(E, A, B)}^{*} \cap \mathscr{W}_{(E, A, B)}^{*}\right)+\operatorname{im} E P_{1}+\operatorname{im} B .
$$

It remains to be shown that the intersection is trivial. To this end, let $x \in\left(E\left(\mathscr{V}_{(E, A, B)}^{*} \cap \mathscr{W}_{(E, A, B)}^{*}\right)+\operatorname{im} B\right) \cap \mathrm{im} E P_{1}$, i.e., $x=E y=E v+b$ for some $y \in \operatorname{im} P_{1}, v \in \mathscr{V}_{(E, A, B)}^{*} \cap \mathscr{W}_{(E, A, B)}^{*}, b \in \operatorname{im} B$. Then $E(y-v)=b$ and hence

$$
y-v \in E^{-1}\{b\} \subseteq E^{-1}(\operatorname{im} B)=\mathscr{W}_{(E, A, B)}^{1} \subseteq \mathscr{W}_{(E, A, B)}^{*} .
$$

This implies $y \in \mathscr{W}^{*} \cap \operatorname{im} P_{1}=\{0\}$ and thus $x=0$.

Step $3 b$ : We show that $l_{2}=n_{2}$. 
We have that

$$
\begin{aligned}
l_{2}=\mathrm{rk} P_{2} & =\operatorname{dim}\left(E \mathscr{V}_{(E, A, B)}^{*}+\operatorname{im} B\right)-\operatorname{dim}\left(\left(E \mathscr{V}_{(E, A, B)}^{*}+\operatorname{im} B\right) \cap\left(A \mathscr{W}_{(E, A, B)}^{*}+\operatorname{im} B\right)\right) \\
& \stackrel{\operatorname{Step} 2 \mathrm{~b}}{=} \operatorname{dim}\left(E \mathscr{V}_{(E, A, B)}^{*}+\operatorname{im} B\right)-\operatorname{dim}\left(E\left(\mathscr{V}_{(E, A, B)}^{*} \cap \mathscr{W}_{(E, A, B)}^{*}\right)+\operatorname{im} B\right) \\
& \stackrel{\text { Step 3a }}{=} \operatorname{dim}\left(\left(E\left(\mathscr{V}_{(E, A, B)}^{*} \cap \mathscr{W}_{(E, A, B)}^{*}\right)+\operatorname{im} B\right) \oplus \operatorname{im} E P_{1}\right)-\operatorname{dim}\left(E\left(\mathscr{V}_{(E, A, B)}^{*} \cap \mathscr{W}_{(E, A, B)}^{*}\right)+\operatorname{im} B\right) \\
& =\operatorname{rk} E P_{1}=\operatorname{rk} P_{1}=n_{2},
\end{aligned}
$$

where $\mathrm{rk} E P_{1}=\operatorname{rk} P_{1}$ follows from the facts that $\operatorname{ker}_{\mathbb{R}} E \subseteq \mathscr{W}_{(E, A, B)}^{*}$ and $\mathscr{W}_{(E, A, B)}^{*} \cap \operatorname{im} P_{1}=\{0\}$.

Step $3 c$ : We show that $E_{22}$ is invertible.

Let $x \in \mathbb{R}^{n_{2}}$ be such that $E_{22} x=0$. Then it follows from (4) that $E P_{1} x=R_{2} E_{12} x$ and hence $E P_{1} x \in \operatorname{im} E P_{1} \cap \operatorname{im} R_{2}=\{0\}$ by Step 3a. This implies $x=0$ since rk $E P_{1}=n_{2}$ by Step $3 b$.

Step 4: We show (iii).

Assume that there is $\lambda \in \mathbb{C}$ and $x \in \mathbb{C}^{n_{3}}$ such that $\left(\lambda E_{33}-A_{33}\right) x=0$. Then (4) implies that

$$
(\lambda E-A) Q_{1} x=R_{2}\left(\lambda E_{13}-A_{13}\right) x+P_{2}\left(\lambda E_{23}-A_{23}\right) x .
$$

Considering the real and imaginary part of the above equation and writing $\lambda=\mu+\mathfrak{i} v, x=x_{1}+\mathfrak{i} x_{2}$ for $\mu, v \in \mathbb{R}$, $x_{1}, x_{2} \in \mathbb{R}^{n}$ we obtain, invoking that $\operatorname{im}\left[R_{2}, P_{2}\right]=E_{(E, A, B)}^{*}+\operatorname{im} B$,

$$
(\mu E-A) Q_{1} x_{1}-v E Q_{1} x_{2} \in E^{\mathscr{V}_{(E, A, B)}^{*}}+\operatorname{im} B \quad \wedge \quad(\mu E-A) Q_{1} x_{2}+v E Q_{1} x_{1} \in E^{\mathscr{V}_{(E, A, B)}^{*}}+\operatorname{im} B .
$$

Hence there exist $v_{1}, v_{2} \in \mathscr{V}_{(E, A, B)}^{*}$ and $b_{1}, b_{2} \in \operatorname{im} B$ such that

$$
(\mu E-A) Q_{1} x_{1}-v E Q_{1} x_{2}=E v_{1}+b_{1}, \quad(\mu E-A) Q_{1} x_{2}+v E Q_{1} x_{1}=E v_{2}+b_{2} .
$$

Then $A Q_{1} x_{1}=E\left(\mu Q_{1} x_{1}-v Q_{1} x_{2}-v_{1}\right)-b_{1}, A Q_{1} x_{2}=E\left(\mu Q_{1} x_{2}+v Q_{1} x_{1}-v_{2}\right)-b_{2}$ and hence

$$
Q_{1} x_{1}, Q_{1} x_{2} \in A^{-1}(\mathrm{im} E+\operatorname{im} B)=\mathscr{V}_{(E, A, B)}^{1} .
$$

Again invoking (5) and noting that both $\mu Q_{1} x_{1}-v Q_{1} x_{2}-v_{1}$ and $\mu Q_{1} x_{2}+v Q_{1} x_{1}-v_{2}$ are contained in $\mathscr{V}_{(E, A, B)}^{1}+$ $\mathscr{V}_{(E, A, B)}^{*}=\mathscr{V}_{(E, A, B)}^{1}$ we obtain

$$
Q_{1} x_{1}, Q_{1} x_{2} \in A^{-1}\left(\mathscr{V}_{(E, A, B)}^{1}+\operatorname{im} B\right)=\mathscr{V}_{(E, A, B)}^{2} .
$$

Repeating this procedure yields $Q_{1} x_{1}, Q_{1} x_{2} \in \mathscr{V}_{(E, A, B)}^{*} \cap \operatorname{im} Q_{1}=\{0\}$ and since $Q_{1}$ has full column rank it follows $x_{1}=x_{2}=0$ and hence $x=0$. Note that the resulting full column rank of $A_{33}$ also implies that $l_{3} \geq n_{3}$. This finishes the proof of the theorem.

Remark 3.2. (i) The full row rank of $\left[E_{11}, B_{1}\right]$ in property (i) of the KCD (3) does not already follow from the complete controllability of $\left(E_{11}, A_{11}, B_{1}\right)$. This is due to the fact, that any completely controllable DAE can be augmented by zero rows (i.e., adding $0=0$ ) without altering its solution behavior at all. However, in the $\mathrm{KCD}$ these zero rows will occur as additional $1 \times 0$ blocks in $\left(E_{33}, A_{33}, 0\right)$. In fact, the $\operatorname{KCD}$ of $\left(\left[\begin{array}{l}1 \\ 0\end{array}\right],\left[\begin{array}{l}1 \\ 0\end{array}\right],\left[\begin{array}{l}1 \\ 0\end{array}\right]\right)$ is obtained with the identity transformations $S=\left[\begin{array}{ll}1 & 0 \\ 0 & 1\end{array}\right]$ and $T=1$, where $l_{1} \times n_{1}=1 \times 1, l_{2} \times n_{2}=0 \times 0$, and $l_{3} \times n_{3}=1 \times 0$.

(ii) In contrast to the above, the addition of a row $0=\tilde{u}$ for some new input $\tilde{u}$, without increasing the state space, does not change the augmented Wong sequences of the overall system. Therefore, these additional rows occur in the block $\left(E_{11}, A_{11}, B_{1}\right)$ in the KCD and lead to the fact that possibly $l_{1}>n_{1}$; for instance the system $\left(\left[\begin{array}{l}1 \\ 0\end{array}\right],\left[\begin{array}{l}0 \\ 1\end{array}\right],\left[\begin{array}{ll}1 & 0 \\ 0 & 1\end{array}\right]\right)$ is already in $\mathrm{KCD}$ with only a $\left(E_{11}, A_{11}, B_{1}\right)$ block. However, it is always true that $l_{1} \leq n_{1}+m$.

(iii) While $l_{2}=n_{2}=0$ just means that the corresponding blocks in the $\mathrm{KCD}$ (3) are not present, we have seen above that $n_{3}=0$ does not imply that the corresponding blocks are not present, they just have zero columns. Analogously, $l_{1}=0$ does also not imply that the corresponding blocks are not present, because there might still 
be $0 \times 1$ blocks present in the KCD (corresponding to free and hence completely controllable variables). For example, the KCD of the DAE ([0], [0], [0]) consists of blocks of the sizes $l_{1} \times n_{1}=0 \times 1, l_{2} \times n_{2}=0 \times 0$ and $l_{3} \times n_{3}=1 \times 0$; in particular, $\operatorname{rk}\left[E_{11}, B_{1}\right]=0=l_{1}$.

(iv) Furthermore, as we have seen in item (ii) above, also $n_{1}=0$ does not imply that the corresponding block in the $\mathrm{KCD}$ is not present, since e.g. the DAE $\left(\left[\begin{array}{l}0 \\ 0\end{array}\right],\left[\begin{array}{l}0 \\ 1\end{array}\right],\left[\begin{array}{l}1 \\ 0\end{array}\right]\right)$ is already in KCD with blocks of sizes $l_{1} \times n_{1}=1 \times 0$, $l_{2} \times n_{2}=0 \times 0$ and $l_{3} \times n_{3}=1 \times 1$; in particular, $\operatorname{rk}\left[E_{11}, B_{1}\right]=\operatorname{rk} B_{1}=1=l_{1}$.

(v) From property (iii) of (3) it follows that $\mathscr{R}_{\left(E_{33}, A_{33}, 0\right)}=\{0\}$ and that $\left(\left[\begin{array}{cc}E_{11} & E_{13} \\ 0 & E_{33}\end{array}\right],\left[\begin{array}{cc}A_{11} & A_{13} \\ 0 & A_{33}\end{array}\right],\left[\begin{array}{c}B_{1} \\ 0\end{array}\right]\right)$ as well as $\left(E_{33}, A_{33}, 0\right)$ are controllable in the behavioral sense. The remaining "uncontrollable" subsystem $\left(E_{22}, A_{22}, 0\right)$ is described by an ODE since $E_{22}$ is invertible. This is remarkable, since the pencil $s E-A \in \mathbb{R}[s]^{l \times n}$ is not necessarily regular.

In the following we prove a uniqueness property of the KCD.

Theorem 3.3 (Uniqueness of KCD). Let $(E, A, B) \in \Sigma_{m}^{l \times n}$ and $S_{1}, S_{2} \in \mathbf{G L}_{l}, T_{1}, T_{2} \in \mathbf{G L}_{n}$ be such that for $i=1,2$

$$
(E, A, B) \stackrel{S_{i}, T_{i}}{=}\left(E_{i}, A_{i}, B_{i}\right)=\left(\left[\begin{array}{ccc}
E_{11, i} & E_{12, i} & E_{13, i} \\
0 & E_{22, i} & E_{23, i} \\
0 & 0 & E_{33, i}
\end{array}\right],\left[\begin{array}{ccc}
A_{11, i} & A_{12, i} & A_{13, i} \\
0 & A_{22, i} & A_{23, i} \\
0 & 0 & A_{33, i}
\end{array}\right],\left[\begin{array}{c}
B_{1, i} \\
0 \\
0
\end{array}\right]\right)
$$

with corresponding block sizes given by $l_{1, i}, n_{1, i}, l_{2, i}, n_{2, i}, l_{3, i}, n_{3, i}$ and the corresponding blocks satisfy conditions (i)(iii) from Theorem 3.1.

Then $l_{1,1}=l_{1,2}, l_{2,1}=l_{2,2}, l_{3,1}=l_{3,1}, n_{1,1}=n_{1,2}, n_{2,1}=n_{2,2}, n_{3,1}=n_{3,2}$ and, moreover, for some $S_{11} \in \mathbf{G L}_{l_{1,1}}, S_{22} \in$ $\mathbf{G L}_{l_{2,1}}, S_{33} \in \mathbf{G L}_{l_{3,1}}, T_{11} \in \mathbf{G L}_{n_{1,1}}, T_{22} \in \mathbf{G L}_{n_{2,1}}, T_{33} \in \mathbf{G L}_{n_{3,1}}$ and $S_{12}, S_{13}, S_{23}, T_{12}, T_{13}, T_{23}$ of appropriate sizes we have that

$$
S_{2} S_{1}^{-1}=\left[\begin{array}{ccc}
S_{11} & S_{12} & S_{13} \\
0 & S_{22} & S_{23} \\
0 & 0 & S_{33}
\end{array}\right], \quad T_{1}^{-1} T_{2}=\left[\begin{array}{ccc}
T_{11} & T_{12} & T_{13} \\
0 & T_{22} & T_{23} \\
0 & 0 & T_{33}
\end{array}\right] \text {. }
$$

In particular,

$$
\left(E_{11,1}, A_{11,1}, B_{1,1}\right) \cong\left(E_{11,2}, A_{11,2}, B_{1,2}\right), \quad\left(E_{22,1}, A_{22,1}, 0\right) \cong\left(E_{22,2}, A_{22,2}, 0\right), \quad\left(E_{33,1}, A_{33,1}, 0\right) \cong\left(E_{33,2}, A_{33,2}, 0\right) .
$$

Proof. Without loss of generality we assume that $S_{1}=I_{l}$ and $T_{1}=I_{n}$.

Step 1: Invoking Remark 2.4 we have

$$
\mathbb{R}^{n_{1,1}} \times\{0\}=\mathscr{R}_{\left(E_{1}, A_{1}, B_{1}\right)}=T_{2} \mathscr{R}_{\left(E_{2}, A_{2}, B_{2}\right)}=T_{2}\left(\mathbb{R}^{n_{1,2}} \times\{0\}\right),
$$

and this implies $n_{1,1}=n_{1,2}$ as well as

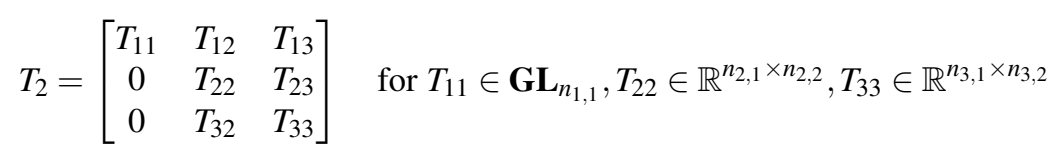

and $T_{12}, T_{13}, T_{23}, T_{32}$ of appropriate size. Furthermore, we have

$$
\mathbb{R}^{n_{1,1}+n_{2,1}} \times\{0\}=\mathscr{V}_{\left(E_{1}, A_{1}, B_{1}\right)}^{*}=T_{2} \mathscr{V}_{\left(E_{2}, A_{2}, B_{2}\right)}^{*}=T_{2}\left(\mathbb{R}^{n_{1,2}+n_{2,2}} \times\{0\}\right),
$$

which together with $n_{1,1}=n_{1,2}$ gives that $n_{2,1}=n_{2,2}, n_{3,1}=n_{3,2}$ and

$$
T_{32}=0, \quad T_{22} \in \mathbf{G L}_{n_{2,1}}, \quad T_{33} \in \mathbf{G L}_{n_{3,1}} .
$$


Step 2: Partitioning

$$
S_{2}=\left[\begin{array}{lll}
S_{11} & S_{12} & S_{13} \\
S_{21} & S_{22} & S_{23} \\
S_{31} & S_{32} & S_{33}
\end{array}\right] \quad \text { for } S_{11} \in \mathbb{R}^{l_{1,2} \times l_{1,1}}, S_{22} \in \mathbb{R}^{l_{2,2} \times l_{2,1}}, S_{33} \in \mathbb{R}^{l_{3,2} \times l_{3,1}},
$$

and off-diagonal block matrices of appropriate size, we find that the equations $S_{2} E_{1} T_{2}=E_{2}$ and $S_{2} B_{1}=B_{2}$ yield that

$$
\left[\begin{array}{l}
S_{21} \\
S_{31}
\end{array}\right]\left[E_{11,1} T_{11}, B_{1}\right]=0
$$

and the full row rank of $\left[E_{11,1}, B_{1}\right]$ then gives $S_{21}=0$ and $S_{31}=0$. Since $S_{2}$ is invertible it follows that $l_{1,1} \leq l_{1,2}$. Reversing the roles of $\left(E_{1}, A_{1}, B_{1}\right)$ and $\left(E_{2}, A_{2}, B_{2}\right)$ gives $l_{1,1} \geq l_{1,2}$, whence $l_{1,1}=l_{1,2}$. We further have the equation

$$
S_{32} E_{22,1} T_{22}=0
$$

which by invertibility of $T_{22}$ and $E_{22,1}$ gives that $S_{32}=0$. This finally implies $l_{2,1}=l_{2,2}=n_{2,1}=n_{2,2}, l_{3,1}=l_{3,2}$, $S_{22} \in \mathbf{G L}_{l_{2,1}}$ and $S_{33} \in \mathbf{G} \mathbf{L}_{l_{3,1}}$ and finishes the proof.

\section{KCD for the regular case}

Regularity of the system $(E, A, B)$ implies that equation (1) has a solution for any (sufficiently smooth) input $u$ and each such solution is uniquely determined by the initial value $x(0)$. Therefore, regularity is often assumed for the analysis and numerical simulation. Due to its importance, we like to highlight some features of the KCD for the regular case. For the solution theory of DAEs, the original Wong sequences (with $B=0$ ) play a fundamental role and we also present the connection between the original Wong sequences and the KCD.

In the following we denote with $\mathscr{V}_{(E, A)}^{*}$ and $\mathscr{W}_{(E, A)}^{*}$ the limits of the original Wong sequences given by

$$
\begin{aligned}
\mathscr{V}_{(E, A)}^{0}:=\mathbb{R}^{n}, & \mathscr{V}_{(E, A)}^{i+1}:=A^{-1}\left(E_{(E, A)}^{i}\right), & i \in \mathbb{N}, \\
\mathscr{W}_{(E, A)}^{0}:=\{0\}, & \mathscr{W}_{(E, A)}^{i+1}:=E^{-1}\left(A \mathscr{W}_{(E, A)}^{i}\right), & i \in \mathbb{N},
\end{aligned}
$$

i.e., $\mathscr{V}_{(E, A)}^{*}=\mathscr{V}_{(E, A, 0)}^{*}$ and $\mathscr{W}_{(E, A)}^{*}=\mathscr{W}_{(E, A, 0)}^{*}$. The original Wong sequences can be used to obtain the quasi-Weierstrass form (QWF) (or quasi-Kronecker form in the singular case $[6,7]$ ).

Proposition 4.1 (Quasi-Weierstrass form, [16], [4]). The DAE (E,A,B) $\in \Sigma_{m}^{n \times n}$ is regular if, and only if,

$$
(E, A, B) \stackrel{S, T}{\cong}\left(\left[\begin{array}{cc}
I & 0 \\
0 & N
\end{array}\right],\left[\begin{array}{ll}
J & 0 \\
0 & I
\end{array}\right],\left[\begin{array}{l}
B_{1} \\
B_{2}
\end{array}\right]\right)
$$

where $N \in \mathbb{R}^{n_{2} \times n_{2}}, 0 \leq n_{2} \leq n$ is nilpotent and $J \in \mathbb{R}^{n_{1} \times n_{1}}, B_{1} \in \mathbb{R}^{n_{1} \times m}, B_{2} \in \mathbb{R}^{n_{2} \times m}, n_{1}:=n-n_{2}$. Furthermore, the transformation matrices $T=\left[T_{1}, T_{2}\right] \in \mathbf{G L}_{n}$ and $S \in \mathbf{G L}_{n}$ achieve the $Q W F(6)$ if, and only if,

$$
\operatorname{im} T_{1}=\mathscr{V}_{(E, A)}^{*}, \quad \operatorname{im} T_{2}=\mathscr{W}_{(E, A)}^{*}, \quad S=\left[E T_{1}, A T_{2}\right]^{-1} .
$$

By Proposition 4.1, the original Wong sequences yield a decoupling of the DAE into an ODE $\dot{v}(t)=J v(t)+B_{1} u(t)$ and a so called pure DAE $N \dot{w}(t)=w(t)+B_{2} u(t)$, where the latter has the unique solution $w=-\sum_{i=0}^{n_{2}-1} N^{i}\left(B_{2} u\right)^{(i)}$; note that by definition of $\mathfrak{B}_{(E, A, B)}$ we only have $u \in \mathscr{L}_{\text {loc }}^{1}$, but $w \in \mathscr{W}_{\text {loc }}^{1}$ and $(w, u)$ being a solution trajectory enforces higher differentiability of the input components $B_{2} u$, see [3, Sec. 2.4.2].

The Wong sequences are coordinate free in the sense that the specific choice of $T_{1}$ and $T_{2}$ is not relevant. Once the QWF is obtained for a specific choice of the coordinate transformation $T$ it is not difficult to obtain a KCD for each block separately (see e.g. [9]): 
Proposition 4.2 (KCD based on QWF). Consider regular $(E, A, B) \in \Sigma_{m}^{n \times n}$. Then

$$
(E, A, B) \cong\left(\left[\begin{array}{cccc}
I & 0 & 0 & 0 \\
0 & I & 0 & 0 \\
0 & 0 & N_{11} & N_{12} \\
0 & 0 & 0 & N_{22}
\end{array}\right],\left[\begin{array}{cccc}
J_{11} & J_{12} & 0 & 0 \\
0 & J_{22} & 0 & 0 \\
0 & 0 & I & 0 \\
0 & 0 & 0 & I
\end{array}\right],\left[\begin{array}{c}
B_{11} \\
0 \\
B_{21} \\
0
\end{array}\right]\right),
$$

where $\left(\left[\begin{array}{cc}I & 0 \\ 0 & N_{11}\end{array}\right],\left[\begin{array}{cc}J_{11} & 0 \\ 0 & I\end{array}\right],\left[\begin{array}{l}B_{11} \\ B_{21}\end{array}\right]\right)$ is completely controllable and $N_{11}$ and $N_{22}$ are nilpotent.

Proof. Assume the DAE $(E, A, B)$ is transformed in QWF (6) with block sizes $n_{1} \times n_{1}$ and $n_{2} \times n_{2}$ and $T=\left[T_{1}, T_{2}\right]$, $S=\left[E T_{1}, A T_{2}\right]^{-1}$. Then choose $T_{11}, T_{12}, T_{21}, T_{22}$ as follows

$$
\begin{aligned}
& \operatorname{im} T_{11}=\operatorname{im}\left\langle J, B_{1}\right\rangle, \quad \operatorname{im}\left\langle J, B_{1}\right\rangle \oplus \operatorname{im} T_{12}=\mathbb{R}^{n_{1},}, \\
& \operatorname{im} T_{21}=\operatorname{im}\left\langle N, B_{2}\right\rangle, \quad \operatorname{im}\left\langle N, B_{2}\right\rangle \oplus \operatorname{im} T_{22}=\mathbb{R}^{n_{2},}
\end{aligned}
$$

where $\langle A, B\rangle=\left[B, A B, A^{2} B, \ldots \ldots A^{n} B\right]$ for $A \in \mathbb{R}^{n \times n}$ and $B \in \mathbb{R}^{n \times m}$. The transformation matrices

$$
T=\left[T_{1}, T_{2}\right]\left[\begin{array}{cccc}
T_{11} & T_{12} & 0 & 0 \\
0 & 0 & T_{21} & T_{22}
\end{array}\right], S=\left(\left[E T_{1}, A T_{2}\right]\left[\begin{array}{cccc}
T_{11} & T_{12} & 0 & 0 \\
0 & 0 & T_{21} & T_{22}
\end{array}\right]\right)^{-1}
$$

then yield the desired KCD, for details see [9].

Clearly, the KCD (7) obtained via the QWF matches the general KCD (3) after a simple rearrangement of the corresponding blocks, in particular

$$
\left(E_{11}, A_{11}, B_{1}\right)=\left(\left[\begin{array}{cc}
I & 0 \\
0 & N_{11}
\end{array}\right],\left[\begin{array}{cc}
J_{11} & 0 \\
0 & I
\end{array}\right],\left[\begin{array}{l}
B_{11} \\
B_{21}
\end{array}\right]\right), \quad\left(E_{22}, A_{22}, 0\right)=\left(I, J_{22}, 0\right), \quad\left(E_{33}, A_{33}, 0\right)=\left(N_{22}, I, 0\right) .
$$

However, the form (7) is not really satisfactory as its derivation needs two separate coordinate transformations: first, one needs to transform the DAE $(E, A, B)$ into QWF and then the ODE and pure DAE parts have to be transformed again. In particular, the latter transformation depends on the chosen coordinate transformation for the QWF (because $J$ and $N$ depend on $T$ ) and is therefore not coordinate free. Furthermore, there is no geometric insight because the connection to the augmented Wong sequences is not clear.

Now we present a more geometric approach. To this end, we need to introduce certain projectors, defined in terms of the Wong sequences, cf. [14].

Definition 4.3 (Consistency, differential and impulse projector). With the notation of Proposition 4.1 define the consistency projector

$$
\Pi_{(E, A)}:=T\left[\begin{array}{ll}
I & 0 \\
0 & 0
\end{array}\right] T^{-1}
$$

the differential projector

$$
\Pi_{(E, A)}^{\text {diff }}:=T\left[\begin{array}{ll}
I & 0 \\
0 & 0
\end{array}\right] S,
$$

and the impulse projector

$$
\Pi_{(E, A)}^{\mathrm{imp}}:=T\left[\begin{array}{ll}
0 & 0 \\
0 & I
\end{array}\right] S,
$$

where the block matrix sizes correspond to the block sizes in the QWF. Furthermore, let

$$
A^{\text {diff }}:=\Pi^{\text {diff }} A, \quad B^{\text {diff }}:=\Pi^{\text {diff }} B, \quad E^{\text {imp }}:=\Pi^{\text {imp }} E, \quad B^{\text {imp }}:=\Pi^{\text {imp }} B .
$$

Note that the consistency projector is a projection onto $\mathscr{V}_{(E, A)}^{*}$ along $\mathscr{W}_{(E, A)}^{*}$, but the differential and impulse projectors are not idempotent and hence are not projectors in the usual sense. Furthermore, it is easy to see that all projectors 
(and consequently $A^{\text {diff }}, B^{\text {diff }}, E^{\text {imp }}, B^{\text {imp }}$ ) do not depend on the specific choice of the transformation matrices $T$ and $S$ (and only on the spaces $\left.\mathscr{V}_{(E, A)}^{*}, \mathscr{W}_{(E, A)}^{*}\right)$. Finally, observe that

$$
\operatorname{im} A^{\text {diff }} \subseteq \mathscr{V}_{(E, A)}^{*}, \operatorname{im} B^{\text {diff }} \subseteq \mathscr{V}_{(E, A)}^{*}, \quad \operatorname{im} E^{\text {imp }} \subseteq \mathscr{W}_{(E, A)}^{*}, B^{\text {imp }} \subseteq \mathscr{W}_{(E, A)}^{*}
$$

With the help of these matrices the connection between the original and augmented Wong sequences can be established as follows.

Theorem 4.4 (Connection between Wong sequences). Let $(E, A, B) \in \Sigma_{m}^{n \times n}$ be regular. Denote with $\mathscr{V}_{(E, A)}^{*}$, $\mathscr{W}_{(E, A)}^{*}$, $\mathscr{V}_{(E, A, B)}^{*}, \mathscr{W}_{(E, A, B)}^{*}$ the limits of the original and augmented Wong sequences, respectively. Using the notation from Definition 4.3, we have

$$
\mathscr{V}_{(E, A, B)}^{*}=\mathscr{V}_{(E, A)}^{*} \oplus \operatorname{im}\left\langle E^{\mathrm{imp}}, B^{\mathrm{imp}}\right\rangle \quad \text { and } \quad \mathscr{W}_{(E, A, B)}^{*}=\mathscr{W}_{(E, A)}^{*} \oplus \operatorname{im}\left\langle A^{\text {diff }}, B^{\text {diff }}\right\rangle .
$$

Proof. Step 1: We show $\mathscr{V}_{(E, A, B)}^{*}=\mathscr{V}_{(E, A)}^{*} \oplus \operatorname{im}\left\langle E^{\mathrm{imp}}, B^{\mathrm{imp}}\right\rangle$.

From Remark 2.4 we know that $\mathscr{V}_{(E, A, B)}^{*}$ equals the space of consistent initial values. On the other hand, from the solution formula in [15, Thm. 6.4.4] it follows that all $(x, u) \in \mathfrak{B}_{(E, A, B)}$ satisfy

$$
x(0)=\Pi_{(E, A)} c-\sum_{i=0}^{v-1}\left(E^{\mathrm{imp}}\right)^{i}\left(B^{\mathrm{imp}} u\right)^{(i)}(0),
$$

for some $c \in \mathbb{R}^{n}$ and $v \in \mathbb{N}$ such that $\left(E^{\text {imp }}\right)^{v}=0$ and $\left(E^{\text {imp }}\right)^{v-1} \neq 0$, where it follows from $x \in \mathscr{W}_{\text {loc }}^{1}$ and [3, Sec. 2.4.2] that $B^{\mathrm{imp}} u \in \mathscr{W}_{\mathrm{loc}}^{v-1}$. Since the derivatives of $B^{\mathrm{imp}} u$ at $t=0$ can be chosen independently of each other it follows that

$$
x_{0} \text { is consistent } \Leftrightarrow x_{0} \in \operatorname{im} \Pi_{(E, A)}+\operatorname{im}\left\langle E^{\mathrm{imp}}, B^{\mathrm{imp}}\right\rangle .
$$

By construction $\operatorname{im} \Pi_{(E, A)}=\mathscr{V}_{(E, A)}^{*}, \operatorname{im}\left\langle E^{\mathrm{imp}}, B^{\mathrm{imp}}\right\rangle \subseteq \operatorname{im} \Pi_{(E, A)}^{\mathrm{imp}} \subseteq \mathscr{W}_{(E, A)}^{*}$ and $\mathscr{V}_{(E, A)}^{*} \cap \mathscr{W}_{(E, A)}^{*}=\{0\}$, hence the claim is shown.

Step 2: We show $\mathscr{W}_{(E, A, B)}^{*}=\mathscr{W}_{(E, A)}^{*} \oplus \operatorname{im}\left\langle A^{\text {diff }}, B^{\text {diff }}\right\rangle$.

First observe that $\mathscr{W}_{(S E T, S A T, S B)}^{*}=T^{-1} \mathscr{W}_{(E, A, B)}^{*}, \mathscr{W}_{(S E T, S A T)}^{*}=T^{-1} \mathscr{W}_{(E, A)}^{*},(S A T)^{\text {diff }}=T^{-1} A^{\text {diff }} T$ and $(S B)^{\text {diff }}=$ $\Pi_{(S E T, S A T)}^{\text {diff }} S B=T^{-1} B^{\text {diff }}$ for any invertible $S$ and $T$; in particular, we have the following equivalences:

$$
\begin{aligned}
\mathscr{W}_{(E, A, B)}^{*}=\mathscr{W}_{(E, A)}^{*} \oplus \operatorname{im}\left\langle A^{\text {diff }}, B^{\text {diff }}\right\rangle & \Leftrightarrow \quad T^{-1} \mathscr{W}_{(E, A, B)}^{*}=T^{-1} \mathscr{W}_{(E, A)}^{*} \oplus T^{-1} \operatorname{im}\left\langle A^{\text {diff }}, B^{\text {diff }}\right\rangle \\
& \Leftrightarrow \quad \mathscr{W}_{(S E T, S A T, S B)}^{*}=\mathscr{W}_{(S E T, S A T)}^{*} \oplus \operatorname{im}\left\langle(S A T)^{\text {diff }},(S B)^{\text {diff }}\right\rangle .
\end{aligned}
$$

Hence we can assume in the following that $(E, A, B)$ is in QWF (6). It is then easy to see that

$$
\mathscr{W}_{(E, A)}^{*}=\{0\} \times \mathbb{R}^{n_{2}} \quad \text { and } \quad \operatorname{im}\left\langle A^{\text {diff }}, B^{\text {diff }}\right\rangle=\operatorname{im}\left\langle J, B_{1}\right\rangle \times\{0\}
$$

and it remains to be shown that

$$
\mathscr{W}_{(E, A, B)}^{*}=\operatorname{im}\left\langle J, B_{1}\right\rangle \times \mathbb{R}^{n_{2}} .
$$

With an inductive argument it is easy to see that

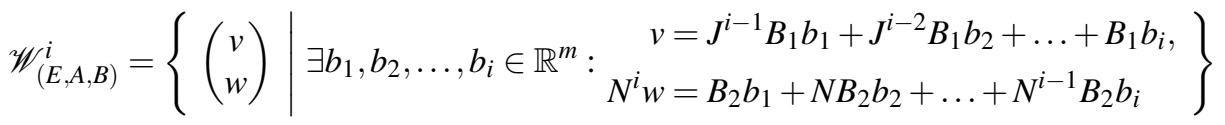

in particular,

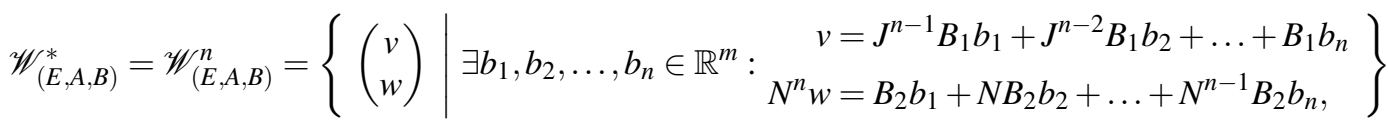




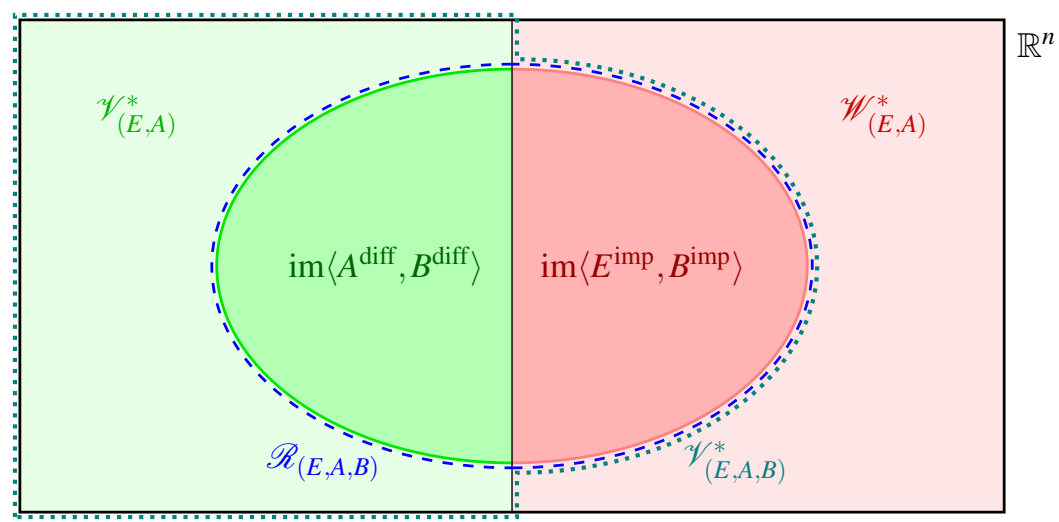

Figure 1: The relationship between the spaces $\mathscr{V}_{(E, A)}^{*}, \mathscr{W}_{(E, A)}^{*}, \operatorname{im}\left\langle A^{\mathrm{diff}}, B^{\mathrm{diff}}\right\rangle, \operatorname{im}\left\langle E^{\mathrm{imp}}, B^{\mathrm{imp}}\right\rangle$.

$$
=\left\{v \mid v=J^{n-1} B_{1} b_{1}+J^{n-2} B_{1} b_{2}+\ldots+B_{1} b_{n},\left(\begin{array}{c}
b_{1} \\
\vdots \\
b_{n}
\end{array}\right) \in \operatorname{ker}\left[B_{2}, N B_{2}, \ldots, N^{n} B_{2}\right]\right\} \times \mathbb{R}^{n_{2}},
$$

because $N^{n}=0$. In fact, it holds that $0=N^{n_{2}}=N^{n_{2}+1}=\ldots=N^{n}$, which implies that $b_{n-n_{1}+1}, b_{n-n_{1}+2}, \ldots, b_{n}$ are free and hence, invoking Cayley-Hamilton, we arrive at

$$
\mathscr{W}_{(E, A, B)}^{*}=\operatorname{im}\left\langle J, B_{2}\right\rangle \times \mathbb{R}^{n_{2}} .
$$

Recalling the observations in Remark 2.4 and the findings in Theorem 4.4, we obtain the following.

Corollary 4.5. With the notation of Theorem 4.4 the following holds:

(i) The consistency space of $(E, A, B)$ is given by $\mathscr{V}_{(E, A, B)}^{*}=\mathscr{V}_{(E, A)}^{*} \oplus \operatorname{im}\left\langle E^{\mathrm{imp}}, B^{\mathrm{imp}}\right\rangle$.

(ii) The reachable space of $(E, A, B)$ is given by $\mathscr{R}_{(E, A, B)}=\mathrm{im}\left\langle A^{\mathrm{diff}}, B^{\mathrm{diff}}\right\rangle \oplus \mathrm{im}\left\langle E^{\mathrm{imp}}, B^{\mathrm{imp}}\right\rangle$, in particular, $(E, A, B)$ is completely controllable if, and only if,

$$
\operatorname{im}\left\langle A^{\mathrm{diff}}, B^{\mathrm{diff}}\right\rangle \oplus \operatorname{im}\left\langle E^{\mathrm{imp}}, B^{\mathrm{imp}}\right\rangle=\mathbb{R}^{n} .
$$

(iii) $(E, A, B)$ is controllable in the behavioral sense if, and only if,

$$
\operatorname{im}\left\langle A^{\mathrm{diff}}, B^{\mathrm{diff}}\right\rangle=\mathscr{V}_{(E, A)}^{*} \quad \text { or, equivalently, } \quad \operatorname{im}\left\langle A^{\mathrm{diff}}, B^{\mathrm{diff}}\right\rangle \oplus \mathscr{W}_{(E, A)}^{*}=\mathbb{R}^{n} .
$$

Proof. Property (i) was already established in the proof of Theorem 4.4. Properties (ii) and (iii) follow directly from Lemma 2.2 and Theorem 4.4 taking into account the following subspace relationships (see also Figure 1):

$$
\operatorname{im}\left\langle A^{\mathrm{diff}}, B^{\mathrm{diff}}\right\rangle \subseteq \mathscr{V}_{(E, A)}^{*} \subseteq \mathscr{V}_{(E, A, B)}^{*}, \quad \operatorname{im}\left\langle E^{\mathrm{imp}}, B^{\mathrm{imp}}\right\rangle \subseteq \mathscr{W}_{(E, A)}^{*} \subseteq \mathscr{W}_{(E, A, B)}^{*}, \quad \mathscr{V}_{(E, A)}^{*} \oplus \mathscr{W}_{(E, A)}^{*}=\mathbb{R}^{n}
$$

Finally, we may obtain the KCD directly in terms of the original system's matrices (and in the original coordinate system) as follows.

Corollary 4.6 (Regular KCD). Use the notation from Definition 4.3 and Theorem 4.4. Choose full column rank matrices $P_{1}, P_{2}, R, Q$ as follows:

$$
\begin{aligned}
& \operatorname{im} P_{1}=\operatorname{im}\left\langle A^{\text {diff }}, B^{\text {diff }}\right\rangle, \quad \operatorname{im}\left\langle A^{\text {diff }}, B^{\text {diff }}\right\rangle \oplus \operatorname{im} R=\mathscr{V}_{(E, A)}^{*}, \\
& \operatorname{im} P_{2}=\operatorname{im}\left\langle E^{\text {imp }}, B^{\text {imp }}\right\rangle, \quad \operatorname{im}\left\langle E^{\text {imp }}, B^{\text {imp }}\right\rangle \oplus \operatorname{im} Q=\mathscr{W}_{(E, A)}^{*} .
\end{aligned}
$$


Then $T=\left[\left[P_{1}, P_{2}\right], R, Q\right] \in \mathbf{G L}_{n}$ and $S=\left[\left[E P_{1}, A P_{2}\right], E R, A Q\right]^{-1} \in \mathbf{G L}_{n}$ transform the DAE $(E, A, B)$ into KCD (3) with some additional zero blocks:

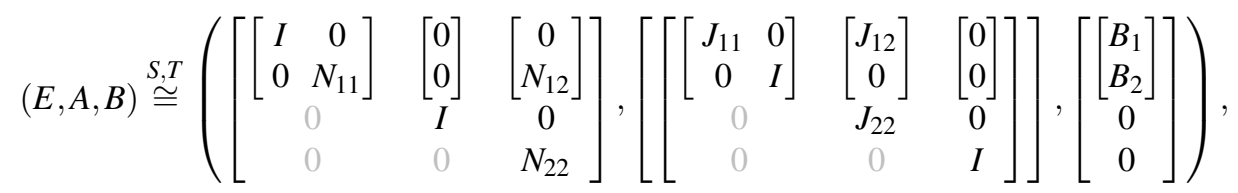

where $\left(\left[\begin{array}{cc}I & 0 \\ 0 & N_{11}\end{array}\right],\left[\begin{array}{cc}J_{11} & 0 \\ 0 & I\end{array}\right],\left[\begin{array}{l}B_{11} \\ B_{21}\end{array}\right]\right)$ is completely controllable and $N_{11}$ and $N_{22}$ are nilpotent.

Proof. Let $\tilde{T}=\left[P_{1}, R, P_{2}, Q\right]$ and $\tilde{S}=\left[E P_{1}, E R, A P_{2}, Q\right]$ be rearranged basis matrices. Then (using the notation from Proposition 4.2)

$$
\begin{aligned}
\tilde{T}^{-1}\left\langle A^{\text {diff }}, B^{\text {diff }}\right\rangle & =\operatorname{im}\left\langle J, B_{1}\right\rangle \times\{0\}=\operatorname{im}\left\langle J_{11}, B_{11}\right\rangle \times\{0\} \subseteq \mathbb{R}^{n_{1}} \times\{0\}, \\
\tilde{T}^{-1}\left\langle E^{\text {imp }}, B^{\text {imp }}\right\rangle & =\{0\} \times \operatorname{im}\left\langle N, B_{2}\right\rangle=\{0\} \times \operatorname{im}\left\langle N_{11}, B_{21}\right\rangle \subseteq\{0\} \times \mathbb{R}^{n_{2}},
\end{aligned}
$$

and the claim follows from Proposition 4.2 with transformation matrices $\tilde{T}$ and $\tilde{S}$.

\section{Conclusion}

We have presented a new Kalman controllability decomposition for general differential-algebraic systems. This decomposition decouples the original DAE into an completely controllable part, a classical uncontrollable part (given by an ODE) and an inconsistent part which is controllable in the behavioral sense but contains no completely controllable part. The corresponding coordinate transformations can easily be obtained via the augmented Wong sequences. For the regular case the construction further simplifies and nice subspace relations become apparent. In particular, a connection between the augmented and the original Wong sequences is established.

\section{References}

[1] Adams, R.A., 1975. Sobolev Spaces. Number 65 in Pure and Applied Mathematics, Academic Press, New York, London.

[2] Banaszuk, A., Kocięcki, M., Przyłuski, K.M., 1990. Kalman-type decompostion for implicit linear discrete-time systems, and its applications. Int. J. Control 52, 1263-1271.

[3] Berger, T., 2014. On differential-algebraic control systems. Ph.D. thesis. Institut für Mathematik, Technische Universität Ilmenau. Universitätsverlag Ilmenau, Ilmenau, Germany.

[4] Berger, T., Ilchmann, A., Trenn, S., 2012. The quasi-Weierstraß form for regular matrix pencils. Lin. Alg. Appl. 436 , $4052-4069$. doi:10.1016/j.laa.2009.12.036.

[5] Berger, T., Reis, T., 2013. Controllability of linear differential-algebraic systems - a survey, in: Ilchmann, A., Reis, T. (Eds.), Surveys in Differential-Algebraic Equations I. Springer-Verlag, Berlin-Heidelberg. Differential-Algebraic Equations Forum, pp. 1-61.

[6] Berger, T., Trenn, S., 2012. The quasi-Kronecker form for matrix pencils. SIAM J. Matrix Anal. \& Appl. 33, 336-368.

[7] Berger, T., Trenn, S., 2013. Addition to "The quasi-Kronecker form for matrix pencils". SIAM J. Matrix Anal. \& Appl. 34, 94-101. doi:10.1137/120883244.

[8] Campbell, S.L., 1980. Singular Systems of Differential Equations I. Pitman, New York.

[9] Dai, L., 1989. Singular Control Systems. Number 118 in Lecture Notes in Control and Information Sciences, Springer-Verlag, Berlin.

[10] Kunkel, P., Mehrmann, V., 2006. Differential-Algebraic Equations. Analysis and Numerical Solution. EMS Publishing House, Zürich, Switzerland.

[11] Polderman, J.W., Willems, J.C., 1998. Introduction to Mathematical Systems Theory. A Behavioral Approach. Springer-Verlag, New York.

[12] Riaza, R., 2008. Differential-Algebraic Systems. Analytical Aspects and Circuit Applications. World Scientific Publishing, Basel.

[13] Rosenbrock, H.H., 1974. Structural properties of linear dynamical systems. Int. J. Control 20, 191-202.

[14] Tanwani, A., Trenn, S., 2010. On observability of switched differential-algebraic equations, in: Proc. 49th IEEE Conf. Decis. Control, Atlanta, USA, pp. 5656-5661.

[15] Trenn, S., 2012. Switched differential algebraic equations, in: Vasca, F., Iannelli, L. (Eds.), Dynamics and Control of Switched Electronic Systems - Advanced Perspectives for Modeling, Simulation and Control of Power Converters. Springer-Verlag, London. chapter 6, pp. 189216.

[16] Weierstraß, K., 1868. Zur Theorie der bilinearen und quadratischen Formen. Berl. Monatsb. , 310-338.

[17] Wong, K.T., 1974. The eigenvalue problem $\lambda T x+S x$. J. Diff. Eqns. 16, 270-280. 